\title{
Challenging Airway Reconstruction: Case Report and Lessons to Be Learned
}

\author{
Eyad Darraj $^{\mathrm{a}}$ Jamal Kassouma $^{\mathrm{a}}$ Yusur Abdulghafoor $^{\mathrm{b}}$ \\ ${ }^{a}$ ENT Department, Dubai Hospital, Dubai, UAE; ${ }^{b}$ Internal Medicine Department, Dubai Health Authority, Dubai, UAE
}

\section{Keywords}

ENT · Medicine $\cdot$ Otolaryngology $\cdot$ Pediatrics $\cdot$ Surgery

\begin{abstract}
Despite the current drop the in incidence rate of acquired subglottic stenosis in comparison to the 1960s and 1970s, this pathology is still encountered and presents a surgical challenge. The wide variety in management options reflects its sophisticated nature and failure tendency. We report a case of a 4-year-old girl who developed severe subglottic stenosis secondary to a 3-month intubation in a leading pediatric hospital and was discharged without any hope of decannulation. The patient was fully investigated in our department, and her successful surgical management ended in the removal of the tracheostomy tube. The complicated surgery and learned lessons urged us to present this case.

(c) 2019 The Author(s)

Published by S. Karger AG, Basel
\end{abstract}

\section{Introduction}

Subglottic stenosis (SGS) is a narrowing of the soft tissue just below the vocal cords. It can be subdivided into congenital and acquired. The acquired type is more commonly encountered than the congenital one, and it is

\begin{tabular}{ll}
\hline KARGER & ( 2019 The Author(s) \\
& Published by S. Karger AG, Basel \\
E-Mail karger@karger.com & This article is licensed under the Creative Commons Attribution- \\
www.karger.com/dmj & NonCommercial-NoDerivatives 4.0 International License (CC BY- \\
NC-ND) (http://www.karger.com/Services/OpenAccessLicense). \\
Usage and distribution for commercial purposes as well as any dis- \\
tribution of modified material requires written permission.
\end{tabular}

mostly iatrogenic in origin. Although the leading cause of acquired SGS is prolonged intubation, it can be a result of trauma or severe infection. There are plenty of management approaches of SGS, endoscopic versus open surgical ones. Tracheostomy is usually needed in severe cases to secure the airway before the definitive treatment.

The aim of this paper is to report a challenging airway reconstruction. However, the most important part is shedding light on the reason that led to airway compromise to avoid it along with its catastrophic consequences.

\section{Case Report}

A 4-year-old, tracheostomized girl with a history of multiple abdominal surgeries and continuous endotracheal intubation for 3 months visited a referral center. The patient developed SGS and underwent tracheostomy. The family visited a leading pediatric center and was informed that "the voice box is extremely scarred, so it is not possible to remove the tracheostomy wherever surgery is carried out." A computed tomography (CT) scan was ordered and showed a subglottic narrowing $21.8 \mathrm{~mm}$ in length with a minimal slit-like luminal diameter of $1.6 \mathrm{~mm}$. It was mainly subglottic with a patent clear trachea (Fig. 1). Direct laryngotracheoscopy under general anesthesia demonstrated severe SGS grade 3 about 2 $\mathrm{cm}$ in length and linear in shape (Fig. 2).

After discussion with the family and exploring the possible treatment options, it was decided to proceed with surgery.

Laryngotracheoplasty was carried out, starting with a graft harvested from the marginal costal cartilage with preservation of the 
1

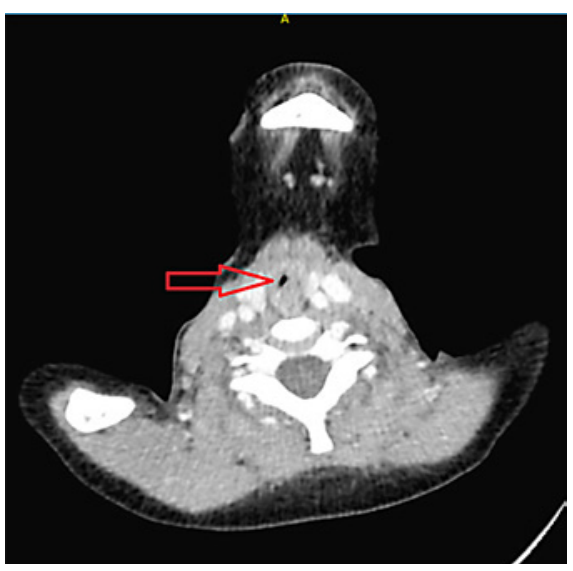

Fig. 1. CT axial view of the slit-like SGS.

Fig. 2. Direct laryngoscopy showing grade 3 SGS.

Fig. 3. Postoperative granulation tissue formation.

Fig. 4. Patent airway with intact mucosa.

3

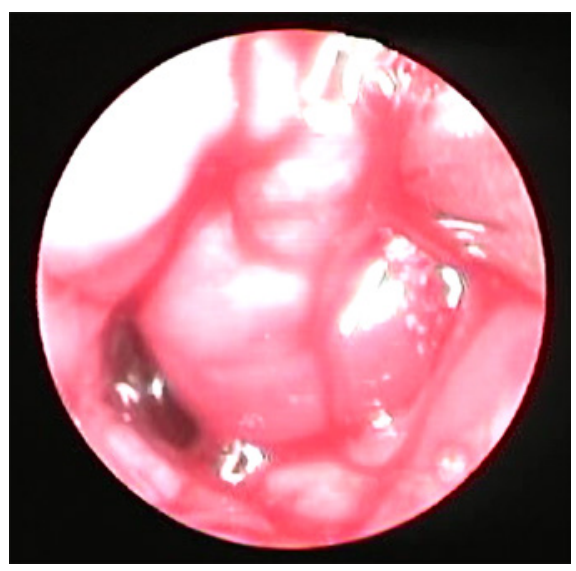

2

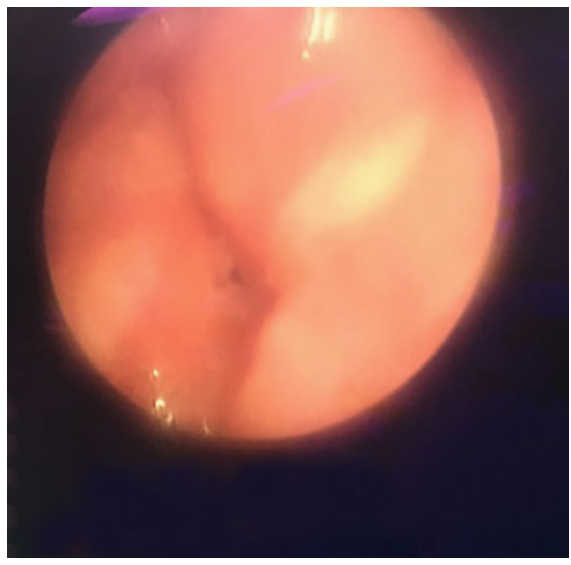

4

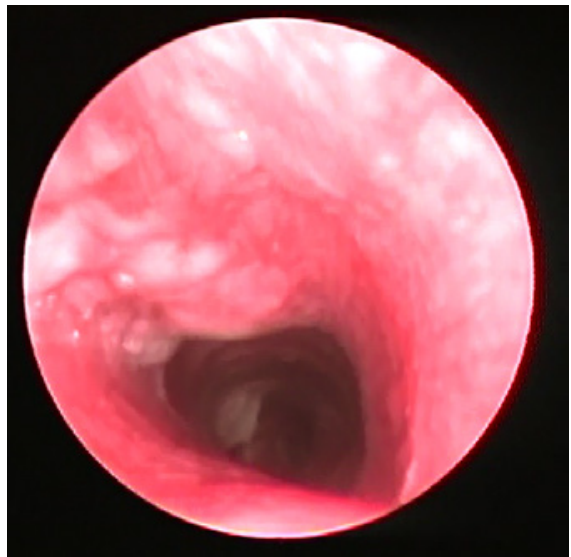

outer perichondrium. The medial perichondrium was kept on the chest to protect the pleura. After a horizontal neck incision above the stoma and subcutaneous dissection, a laryngotracheal fissure was done to involve the lower half of the thyroid cartilage, cricoid, and upper three rings of the trachea. Due to severe SGS (grade 3), the decision was to split the posterior cricoid lamina and to fix the graft to the anterior wall of the trachea with its perichondrium towards the new lumen. A tracheostomy tube No. 5 was used as a stent and fixed by a Prolene suture at the bottom.

Three months later, the stent was removed under general anesthesia. The graft was intact, but extensive granulation tissue was noted (Fig. 3). This was managed by high-dose antibiotics and a steroid regime. One week later, endoscopy was carried out using a rigid endoscope and showed patent airways up to the carina with normal mucosa (Fig. 4). After serial episodes of endoscopic assessment showing a wide subglottic area, the decannulation process started. On a regular follow-up, the patient was found to do well, eating and breathing normally even during play and exercise. At the time of writing this article, 15 months had passed since decannulation, and the patient has not reported any symptoms of breathing difficulties.

Challenging Airway Reconstruction

\section{Discussion}

The subglottic area of the larynx houses the cricoid cartilage, which is the only complete cartilage along the airways. This cartilage does not expand and lacks flexibility, making the subglottic area the narrowest part of the airways.

SGS could be congenital or acquired, and the vast majority of SGS cases in the 19th century were due to specific infections such as syphilis, tuberculosis, diphtheria, and others.

In the 1960s and 1970s, the incidence of acquired SGS increased significantly. It is thought that this was due to the increased practice of prolonged neonatal intubation. This situation urged surgeons to study the relations between intubation and stenosis incidence and to look for new techniques to manage this condition $[1,2]$.

In the 1990s, the incidence of SGS dropped dramatically. A report from the Children's National Medical 
Center in Washington published in 1998 showed that only $1-2 \%$ of the children admitted to the NICU developed SGS [3].

It has been observed that endotracheal intubation causes injury and remarkable changes in the lining mucosa and perichondrium of the cricoid cartilage, starting with edema and congestion that may develop to microscopic necrosis. With prolonged intubation, further perichondrium exposure could happen. A fibrotic scar results in case of infection upon the exposed area. These events are accelerated when a larger tube is used.

SGS usually manifests with biphasic stridor; however, glottic and SGS might present in the same way. Indication of surgical intervention is based upon the severity of symptoms rather than the grade of stenosis. Myers and Cotton [4] classified this stenosis into four grades according to the extension of obstruction:

Grade I $-0-50 \%$ of the lumen is obstructed

Grade II $-51-70 \%$ of the lumen is obstructed

Grade III $-71-99 \%$ of the lumen is obstructed

Grade IV - $100 \%$ of the lumen is obstructed

Often, grades I and II do not require surgery. This is also the case when the symptoms are sporadic and can be treated medically. However, when symptoms persist, and the frequency or severity of stridor increases, surgery should be considered.

There is no absolute contraindication to airway reconstruction as long as the child is fit for general anesthesia. A relative contraindication is when the child needs tracheostomy for another reason (e.g., lower airway toilet), and severe gastroesophageal reflux disease (GERD) is a relative contraindication as well.

Endoscopic assessment is the cornerstone in the evaluation of airway obstruction. Flexible fiberoptic nasoendoscopy can be tried first. It is a simple, office-based procedure with the advantage of vocal cord mobility assessment; however, its visualization of the subglottic area is not optimal.

Rigid endoscopy under general anesthesia is superior to flexible endoscopy in the assessment of laryngeal stenosis and the bronchial tree. It is of paramount importance to have a full set of instruments during the procedure (laryngoscopes, bronchoscopes, telescopes, camera, light source, etc.) and to maintain proper communication with the anesthesiologist. The assessment should include the supraglottis, glottis, and subglottis, looking for edema, granuloma, and signs of GERD. The surgeon should also document the length, thickness, and maturity of stenosis in addition to stage as per Cotton-Myer classification [4].
Radiologic studies usually start with a plain X-ray of the neck (anteroposterior and lateral views), and SGS appears peaked with the pathognomonic "steeple" sign. Barium swallow might be requested to rule out GERD and vascular abnormalities, keeping CT and magnetic resonance imaging for the more advanced and complicated cases.

The treatment of SGS could start medically. If the stenosis is in an early stage and is not fibrosed yet, oral or inhaler steroids can be helpful during this period by managing inflammation. Once the stenosis gets scarred and becomes mature, medical treatment is ineffective. However, treatment of GERD is mandatory even when surgical intervention is planned.

There is a wide spectrum of surgical interventions, ranging from endoscopic procedures to open reconstruction to cricotracheal resection. Endoscopic procedures are often indicated in mild and immature stenosis. Quesnel et al. [5] described the usage of $\mathrm{CO}_{2}$ laser endoscopically in circumferential subglottic cases by making four incisions followed by dilatation. Some surgeons use medications in endoscopic procedures: steroid injection and/or topical mitomycin C application.

Mitomycin is antiproliferative, working by blocking fibroblast division and activity. In 2005, Hueman and Simpson [6] studied the side effects of mitomycin application and found that $4.7 \%$ of the cases got fibrin debris at the surgical site, and no significant systemic adverse effects.

Balloon dilatation is another tool that can be used endoscopically. However, the outcomes in advanced stages (grades 3 and 4) are far less successful than open reconstruction [7].

Open surgical approaches are often tailored as per extension of the stenosis, thickness of the scar, and status of the surrounding tissue. The aim of these surgeries is to decannulate the child and preserve his/her voice.

In 1980, anterior cricoid split was first described by Cotton and Seid [8]. It decompresses subglottic edema and gives more room to expand the airways. Possible complications of anterior cricoid split are infection, surgical emphysema, pneumothorax, and expansion failure.

In 1991, laryngotracheoplasty was first described by Seid et al. [9]. It includes fixation of part of the cartilage into the split area instead of leaving it to fibrosis. Expanding the laryngeal lumen using cartilage has significantly increased the chance of extubation. This was confirmed by Richardson [10] who compared the success rate when a cartilage graft was used (90\%) versus leaving 
the split area without cartilage (56\%) in infants $<6$ months with acquired stenosis.

For more complicated and advanced stages of stenosis, further grafting of the posterior lamina of the cricoid cartilage was suggested. Decannulation was successful in more than $90 \%$ of the operated cases using this method, with rare need for further surgery as Zalzal and Cotton [11] reported.

Often, during tracheostomy a stent is placed that lasts for 4-6 weeks. After stent removal, endoscopic assessment of the airways using a rigid endoscope is mandatory before starting the process of decannulation.

Even with anterior-posterior reconstructions, some advanced stages of stenosis have failed to maintain adequate lumen. Monnier from Switzerland took a remarkable step in airway surgery by the introduction of cricotracheal resection with a successful decannulation rate of $97 \%$ in 31 children.

This surgery is indicated in patients with severe SGS, free glottis, and a minimum of $4 \mathrm{~mm}$ healthy mucosa between the vocal cords and stenosis. Due to expected edema, the airway should be secured with tracheostomy or a T tube for 4-6 weeks until all edema subsides.

Follow-up bronchoscopy is often done within 1-3 weeks after surgery to evaluate the patency and restenosis of the airways.

Zalzal et al. [12] studied the etiology of failed reconstruction in 27 cases and found that reasons for failure are (a) improper selection of a graft and/or stent; (b) the stent gets removed too early; (c) poor preoperative evaluation and postoperative care; (d) active reflux; and (e) keloid.

Complications of airway reconstructions are wound infection, pneumonia, atelectasis, surgical emphysema, pneumothorax, and pneumomediastinum.

Outcomes of airway reconstruction are mainly determined by the severity of stenosis and the surgery performed. Generally, the successful decannulation rate is about $80-90 \%$ when the surgery and patient are properly selected.

Choi and Zalzal [13] reported that the age of the child is an important factor in failed reconstruction; children $<2$ years are at a higher risk for subglottic scars than others.

It is thought that the surgical options of airway reconstruction have been explored, and now it is time to investigate absorbable stents and to study new medications that can interfere with growth factors and prevent restenosis.

\section{Conclusion}

The catastrophic situation of the girl presented herein resulted from prolonged intubation without suggestion for tracheostomy. So, it is important to consider tracheostomy whenever long-term ventilation is planned, with selection of a proper tube and good fixation.

We are not sure if our patient's body reacted to our endotracheal tube or the tube was moving, resulting in granulation tissue. Since the trachea was full of granulation tissue, particularly on the posterior wall, the decision was made to try high-dose antibiotics and steroids to avoid an aggressive surgery that might have resulted in tracheoesophageal fistula.

Fortunately, the treatment of our patient was successful and did not require further surgery. So, it is a very good alternative that could also work in similar cases. Careful investigation and a proper management plan led to a favorable result. Hence, our patients deserve all our efforts and passion without losing hope.

\section{Statement of Ethics}

Consent was given by the patient.

\section{Disclosure Statement}

The authors have no conflicts of interest to disclose.

\section{Funding Sources}

The authors did not receive any funding.

\section{Author Contributions}

All authors contributed equally.

\section{References}

1 Manica D, Schweiger C, Maróstica PJ, Kuhl G, Carvalho PR. Association between length of intubation and subglottic stenosis in children. Laryngoscope. 2013 Apr;123(4):1049-54.

2 Schweiger C, Marostica PJ, Smith MM, Manica D, Carvalho PR, Kuhl G. Incidence of post-intubation subglottic stenosis in children: prospective study. J Laryngol Otol. 2013 Apr;127(4):399-403.

3 Choi SS, Zalzal GH. Changing trends in neonatal subglottic stenosis. Otolaryngol Head Neck Surg. 2000 Jan;122(1):61-3. 
4 Myer CM 3rd, Cotton RT. Historical development of surgery for pediatric laryngeal stenosis. Ear Nose Throat J. 1995 Aug;74(8):560-2, 564.

5 Quesnel AM, Lee GS, Nuss RC, Volk MS, Jones DT, Rahbar R. Minimally invasive endoscopic management of subglottic stenosis in children: success and failure. Int J Pediatr Otorhinolaryngol. 2011 May;75(5):652-6.

6 Hueman EM, Simpson CB. Airway complications from topical mitomycin C. Otolaryngol Head Neck Surg. 2005 Dec;133(6):831-5.
7 Maresh A, Preciado DA, O'Connell AP, Zalzal GH. A comparative analysis of open surgery vs endoscopic balloon dilation for pediatric subglottic stenosis. JAMA Otolaryngol Head Neck Surg. 2014 Oct;140(10):901-5.

8 Cotton RT, Seid AB. Management of the extubation problem in the premature child. Anterior cricoid split as an alternative to tracheotomy. Ann Otol Rhinol Laryngol. 1980 NovDec;89(6 Pt 1):508-11.

9 Seid AB, Pransky SM, Kearns DB. One-stage laryngotracheoplasty. Arch Otolaryngol Head Neck Surg. 1991 Apr;117(4):408-10.

10 Richardson MA, Inglis AF Jr. A comparison of anterior cricoid split with and without costal cartilage graft for acquired subglottic stenosis. Int J Pediatr Otorhinolaryngol. 1991 Sep;22(2):187-93.
11 Zalzal GH, Cotton RT. A new way of carving cartilage grafts to avoid prolapse into the tracheal lumen when used in subglottic reconstruction. Laryngoscope. 1986 Sep;96(9 Pt 1): 1039.

12 Zalzal GH, Choi SS, Patel KM. Ideal timing of pediatric laryngotracheal reconstruction. Arch Otolaryngol Head Neck Surg. 1997 Feb; 123(2):206-8.

13 Choi SS, Zalzal GH. Pitfalls in laryngotracheal reconstruction. Arch Otolaryngol Head Neck Surg. 1999 Jun;125(6):650-3. 